\title{
On Stability Analysis of Finite Difference Schemes for Generalized Kuramoto-Tsuzuki Equation with Nonlocal Boundary Conditions
}

\author{
Teresè Leonavičienè $\dot{x}^{a}$, Andrej Bugajev ${ }^{a}$, \\ Gerda Jankevičiūtè ${ }^{a}$ and Raimondas Čiegis ${ }^{a}$ \\ ${ }^{a}$ Vilnius Gediminas Technical University \\ Saultekio al. 11, LT-10223 Vilnius, Lithuania \\ E-mail(corresp.): terese.leonaviciene@vgtu.lt \\ E-mail: andrej.bugajev@vgtu.1t \\ E-mail: gerda.jankeviciute@vgtu.lt \\ E-mail: raimondas.ciegis@vgtu.lt
}

Received February 10, 2016; revised May 28, 2016; published online September 15, 2016

\begin{abstract}
A general methodology for the stability analysis of discrete approximations of nonstationary PDEs is applied to solve the Kuramoto-Tsuzuki equation, including also the Schrödinger problem. Stability regions are constructed for the explicit, backward and symmetrical Euler schemes. The obtained results are applied to solve the Kuramoto-Tsuzuki problem with a non-local integral boundary condition. Results of computational experiments are provided.
\end{abstract}

Keywords: finite difference method, stability analysis, Kuramoto-Tsuzuki equation, nonlocal boundary conditions.

AMS Subject Classification: 65N06; 65N12; 35Q56.

\section{Introduction}

Non-classical and nonlocal boundary conditions are used in various real-world applications, e.g. parabolic problems in heat conduction and thermodynamics $[2,6,10]$, and pseudo-parabolic problems in underground water flow $[1,9]$, see also references given in these papers. It is well-known that the well-posedness and stability of such models depends essentially on specific boundary conditions.

Another important topic is a numerical approximation of such problems and the stability analysis of obtained discrete problems. The stability analysis of parabolic and pseudoparabolic problems with nonlocal boundary conditions is done by various methods. A short review of these methods is presented in [7].

In papers $[5,7]$, we have proposed a methodology, when the stability of the specified model is investigated in two steps, and the influence of the differential 
equation (and a finite-difference or finite-volume scheme) is separated from the analysis of the influence of nonlocal boundary conditions. Thus a big part of existing stability results can be reused if a new type of mathematical model with nonlocal boundary conditions is analysed. A short description of this methodology will be given in the next section.

In this paper, as a basic mathematical model we consider the one dimensional Kuramoto-Tsuzuki equation [17]:

$$
\frac{\partial u}{\partial t}=\left(1+i c_{0}\right) u+\left(1+i c_{1}\right) \frac{\partial^{2} u}{\partial x^{2}}-\left(1+i c_{2}\right)|u|^{2} u
$$

where $t$ and $x$ are time and space variables, $c_{0}, c_{1}, c_{2}$ are real constants and $u=u(x, t)$ is unknown complex-valued function. It describes a dynamics of chemical reaction-diffusion systems. A similar equation (1.1) is considered in the analysis of nonlinear optics and fluid dynamics processes, described by the Ginzburg-Landau equation [16].

The Kuramoto-Tsuzuki and Ginzburg-Landau problems with basic classical boundary conditions are solved numerically by using various methods, e.g. the finite difference method with the explicit and symmetrical Euler integration in time [20, 21, 26, 27] and the pseudospectral method [29]. A mixture of implicit and semi-explicit integration techniques are used in $[3,28]$. The stability analysis is done in the specified energy norms and uniform error estimates are proved by using embedding theorems.

The main goal of this paper is to investigate the stability regions of some standard time-integration methods for a linear part of the Kuramoto-Tsuzuki problem. Nonlocal boundary conditions are considered, thus the eigenvalues of the obtained operators can be complex numbers. We consider a linear equation

$$
\frac{\partial u}{\partial t}=\left(c_{R}+i c_{I}\right) \frac{\partial^{2} u}{\partial x^{2}}
$$

where $c_{R}, c_{I}$ are real constants. Note, that equation (1.2) is a very useful model, since it generalizes two important cases of evolutionary equations. Taking $c_{I}=0, c_{R}>0$ we get a parabolic equation (which simulates various diffusion processes) and taking $c_{R}=0$ we get the Schrödinger equation (which is a basis for most nonlinear optics and quantum mechanics models). The stability and convergence of numerical schemes for parabolic and Schrödinger problems are investigated quite well, but a general case of complex coefficients $c_{R}+i c_{I}$, $c_{R}>0$ still requires a proper analysis.

Let equation (1.2) is solved in the interval $D=(0,1)$. We formulate an initial condition

$$
u(x, 0)=\phi(x), \quad 0<x<1
$$

and boundary conditions:

$$
u(0, t)=0, \quad u(1, t)=\gamma \int_{0}^{1} u(x, t) d x, \quad t>0,
$$

where $\phi$ is a known function and $\gamma$ is a given constant. The second boundary condition is an example of nonlocal boundary conditions. 
Next we give a brief review of recent stability analysis methods used for investigation of numerical approximations of parabolic and pseudoparabolic equations with nonlocal boundary conditions.

For many nonlinear problems the main aim is to prove stability estimates in the maximum norm. Here we mention a general technique based on a presentation of the solution of a given PDE with nonlocal boundary conditions as a superposition of solutions of classical boundary value problems. For stationary problems, this method is applied in [4], for non-stationary problems in $[6,10]$ (see references contained therein). Still it is not clear if the maximum principle can be applied for the analysis of numerical approximations of pseudo-parabolic problems with nonlocal conditions.

For the Galerkin, finite volume and finite difference space discretization schemes with standard time integration approximations (e.g. the Crank-Nicolson method) the stability analysis is done by applying the energy estimates, see $[9,11,18]$ and references contained therein. Note, that in order to apply energy estimates we should answer the question how to select the right norm or functional for the given problem (including nonlocal boundary conditions).

One general technique to prove necessary and sufficient stability conditions for non-stationary numerical approximations of differential problems is to apply the eigenvalue criterion for non-normal matrices $[7,13]$. This stability analysis technique is applied for simple parabolic and pseudo-parabolic problems with nonlocal boundary conditions in $[12,14]$. A structure of the spectrum of discrete operators with various nonlocal boundary conditions is investigated in $[15,24]$.

The rest of this paper is organized as follows. In Section 2, the details of a general stability analysis methodology are presented. The stability region for the Kuramoto-Tsuzuki differential equation is constructed. Finite difference schemes are investigated in Section 3. The stability regions are constructed for the explicit, backward and symmetrical Euler schemes. The obtained results are adapted for the case, when all eigenvalues of the discrete operator are real. A review on structure of eigenvalues for some nonlocal boundary conditions is described in Section 4. Results of numerical experiments are given in Section 5. They illustrate the theoretical results. Some final conclusions are presented in Section 5.

\section{The methodology of stability analysis}

Here we apply the same stability analysis template as in $[5,7]$. The main idea is to split the stability analysis of the given differential equation (or time integration method) and the analysis of a structure of the spectrum for a specified discrete operator with various nonlocal boundary conditions.

This methodology consists of two steps. We describe it for the fully discrete numerical approximation of the differential problem. First, the stability of a numerical time integration method is investigated. This analysis should be done only once and the result is defined in a form of simple stability conditions. An example of such conditions can be the stability region in complex space. Second, eigenvalues of discrete operators of interest (including specific 
boundary conditions) should be determined and then the stability conditions of the given integration method can be applied.

In the case of nonlocal boundary conditions, the matrix of discrete operator $A_{h}$ is non-normal. The spectral stability analysis is still can be used for such operators if these matrices can be diagonalized

$$
\mathcal{A}_{h}=\Phi \Lambda(\Phi)^{-1}, \quad \Lambda=\operatorname{diag}\left(\lambda_{j}\right), \quad j=1, \ldots, J
$$

and eigenvectors $\Phi$ make a complete basis system. In our paper, we always assume that this property is valid.

In this section we construct the stability region of the Kuramoto-Tsuzuki differential equation (1.2). Let us write this equation in the operator form

$$
\frac{\partial u}{\partial t}=\left(c_{R}+i c_{I}\right) A u, \quad(x, t) \in D \times(0, T]
$$

where

$$
A u=\frac{\partial^{2} u}{\partial x^{2}}
$$

and its definition also includes boundary conditions. Let $\varphi_{j}, \lambda_{j}$ be eigenfunctions and eigenvalues of operator $A$ :

$$
A \varphi_{j}=\lambda_{j} \varphi_{j}
$$

In general, eigenvalues $\lambda_{j}$ are complex numbers $\lambda_{j}=\lambda_{j R}+i \lambda_{j I}$.

As it was stated above, we assume that these eigenfunctions are linearly independent and make a complete basis system, thus the solution $u(x, t)$ can be written in a form:

$$
u(x, t)=\sum_{j} c_{j}(t) \varphi_{j}(x) .
$$

Inserting this sum into differential equation (2.1) we obtain linear ODEs for functions $c_{j}$ :

$$
c_{j}^{\prime}(t)=\left(c_{R}+i c_{I}\right)\left(\lambda_{j R}+i \lambda_{j I}\right) c_{j}(t), \quad j=1,2 \ldots,
$$

so that

$$
c_{j}(t)=e^{\tilde{\lambda}_{j} t} c_{j}(0), \quad \tilde{\lambda}_{j}=\left(\lambda_{j R}+i \lambda_{j I}\right)\left(c_{R}+i c_{I}\right) .
$$

Then the standard conclusion follows, that differential problem (2.1) is stable if $\operatorname{Re} \widetilde{\lambda}_{j} \leq 0$ [13]. After simple computations we find that the stability region of the Kuramoto-Tsuzuki problem is defined by the inequality

$$
c_{R} \lambda_{R}-c_{I} \lambda_{I} \leq 0
$$

From (2.2) it follows straightforwardly that the stability region of a parabolic equation $\left(c_{R} \geq 0, c_{I}=0\right)$ is defined by the inequality

$$
\lambda_{R} \leq 0,
$$




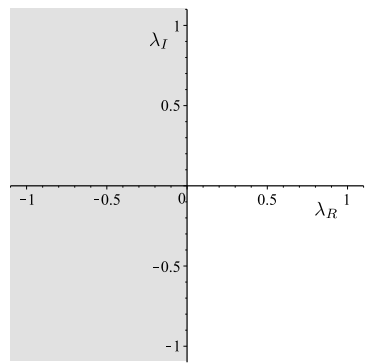

(a) parabolic equation

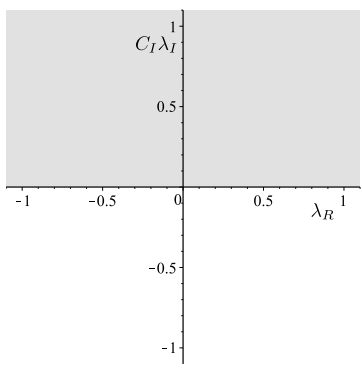

(b) Schrödinger equation



(c) Kuramoto-Tsuzuki equation

Figure 1. Stability regions of a) parabolic equation, b) Schrödinger equation, c) Kuramoto-Tsuzuki equation

or the entire left complex half-plane. The stability region of the Schrödinger equation $\left(c_{R}=0\right)$ is defined by the inequality

$$
c_{I} \lambda_{I} \geq 0
$$

or the entire upper complex half-plane. These stability regions are presented in Fig. 1.

As a conclusion we note that for all three considered equations stability regions define complex half-plains rotated according to a new orthogonal coordinate system

$$
\widetilde{\lambda}_{R}=c_{R} \lambda_{R}-c_{I} \lambda_{I}, \quad \widetilde{\lambda}_{I}=c_{I} \lambda_{R}+c_{R} \lambda_{I},
$$

where the angle of rotation depends on coefficients $c_{R}, c_{I}$ of the equation.

\section{$3 \quad$ Finite Difference Schemes}

In this section we approximate Kuramoto-Tsuzuki equation (1.2) by the standard Euler finite-difference schemes. The domain $\bar{D}$ is covered by the discrete uniform grid

$$
\bar{D}_{h}=\left\{x_{j}: x_{j}=j h, \quad j=0, \ldots, J\right\}, \quad x_{J}=1,
$$

$\bar{D}_{h}=D_{h} \cup \partial D_{h}$. Let $\omega_{\tau}$ be a uniform time grid

$$
\omega_{\tau}=\left\{t^{n}: t^{n}=n \tau, \quad n=0, \ldots, N, N \tau=T\right\},
$$

where $\tau$ is the time step. Although the constant time step $\tau$ is taken here, the following studies can be easily extended to the case when $\tau$ varies.

We consider numerical approximations $U_{j}^{n}$ to the exact solution $u_{j}^{n}=$ $u\left(x_{j}, t^{n}\right)$ at the grid points $\left(x_{j}, t^{n}\right) \in \bar{D}_{h} \times \omega_{\tau}$. For functions defined on the 
grid we introduce the forward and backward difference quotients with respect to $x$

$$
\partial_{x} U_{j}^{n}=\left(U_{j+1}^{n}-U_{j}^{n}\right) / h, \quad \partial_{\bar{x}} U_{j}^{n}=\left(U_{j}^{n}-U_{j-1}^{n}\right) / h
$$

and similarly the backward difference quotient and the averaging operator with respect to $t$

$$
\partial_{\bar{t}} U_{j}^{n}=\left(U_{j}^{n}-U_{j}^{n-1}\right) / \tau, \quad U_{j}^{n-1+\theta}=\theta U_{j}^{n}+(1-\theta) U_{j}^{n-1} .
$$

We approximate the differential equation (1.2) by the finite difference scheme

$$
\partial_{\bar{t}} U_{j}^{n}=\left(c_{R}+i c_{I}\right) A_{h} U_{j}^{n-1+\theta} \quad x_{j} \in D_{h}, \quad n>0
$$

where discrete operator $A_{h}$ is defined as

$$
A_{h} U=\partial_{x} \partial_{\bar{x}} U, \quad x \in D_{h}
$$

and specific boundary conditions will be added later. Here $\theta=0$ defines the explicit Euler method, $\theta=1$ defines the backward Euler method and $\theta=1 / 2$ gives the symmetric (or trapezoidal) Euler method [13]. At the moment we are not specifying any boundary conditions.

Next we apply the same stability analysis methodology as was described for the differential equation case. Let $\varphi_{h j}, \lambda_{h j}$ be eigenvectors and eigenvalues of discrete operator $A_{h}$ :

$$
A_{h} \varphi_{h j}=\lambda_{h j} \varphi_{h j}
$$

In general (e.g. for nonlocal boundary conditions), eigenvalues $\lambda_{h j}$ are complex numbers $\lambda_{h j}=\lambda_{h j R}+i \lambda_{h j I}$.

As it was stated above, we assume that these eigenvectors are linearly independent and make a complete basis system. Then the stability region of finite difference scheme is determined by investigating solutions of the scalar linear equation

$$
\frac{U^{n}-U^{n-1}}{\tau}=\left(c_{R}+i c_{I}\right)\left(\lambda_{h R}+i \lambda_{h I}\right)\left(\theta U^{n}+(1-\theta) U^{n-1}\right) .
$$

We formulate the standard characteristic equation

$$
\frac{q-1}{\tau}=\left(\widetilde{\lambda}_{h R}+i \widetilde{\lambda}_{h I}\right)(\theta q+1-\theta)
$$

where $\tilde{\lambda}$ is defined by $(2.3)$. The stability regions for all three Euler schemes are well-known [13]. Let us denote $\widetilde{\mu}_{h}=\tau \widetilde{\lambda}_{h}$. The stability region of the explicit Euler scheme is defined by the condition

$$
\left|\widetilde{\mu}_{h}+1\right| \leq 1
$$

the stability region of the backward Euler scheme is given by

$$
\left|\widetilde{\mu}_{h}-1\right| \geq 1
$$


and the stability region of the symmetric Euler scheme is given by the entire left complex half-plane:

$$
\widetilde{\mu}_{h R} \leq 0 .
$$

Next we map these standard stability regions into a basic complex plane $\left(\lambda_{R}, \lambda_{I}\right)$. Taking into account the relation (2.3) between two orthogonal coordinate systems, we get that the respective stability regions are rotated by some angle and scaled by factor $1 / \sqrt{c_{R}^{2}+c_{I}^{2}}$.

The stability region of the explicit Euler scheme is defined by the condition

$$
\left(c_{R} \mu_{h R}-c_{I} \mu_{h I}+1\right)^{2}+\left(c_{I} \mu_{h R}+c_{R} \mu_{h I}\right)^{2} \leq 1 .
$$

It is interesting to note, that for the Schrödinger equation, when $c_{R}=0$, this region do not include any interval of real eigenvalues. Thus in the case of classical boundary conditions when all eigenvalues of the discrete operator $A_{h}$ are real, the explicit Euler integration scheme is unconditionally unstable.

The stability region of the backward Euler scheme is defined by the condition

$$
\left(c_{R} \mu_{h R}-c_{I} \mu_{h I}-1\right)^{2}+\left(c_{I} \mu_{h R}+c_{R} \mu_{h I}\right)^{2} \geq 1
$$

and the stability region of the symmetric Euler scheme is given by the rotated entire left complex half-plane:

$$
c_{R} \lambda_{h R}-c_{I} \lambda_{h I} \leq 0 .
$$

This results shows that the symmetric Euler scheme is $A(\alpha)$-stable [13]. We note, that the stability region of the discrete symmetric Euler algorithm exactly coincides with the stability region of the parabolic, Schrödinger and KuramotoTsuzuki equations.

Examples of stability regions of the explicit Euler scheme for approximations of different differential equations and scaling coefficients $c_{R}, c_{I}$ are presented in Fig. 2.

Having exact information on stability regions of different time integration schemes we can adapt these results to numerical analysis of important applications. Let us consider the stability region of the explicit Euler algorithm (3.1) and assume that all eigenvalues of the discrete operator $A_{h}$ are real, i.e. $\mu_{h I}=0$. Then the stability line on real axis is defined by

$$
\left(c_{R} \mu_{h R}+1\right)^{2}+\left(c_{I} \mu_{h R}\right)^{2} \leq 1
$$

the solution of this inequality is

$$
\mu_{h R} \in\left[-\frac{2 c_{R}}{c_{R}^{2}+c_{I}^{2}}, 0\right] .
$$

For the Kuramoto-Tsuzuki equation with parameters as in Figure 2(c) the explicit Euler scheme is stable when $\mu_{h R} \in\left[-\frac{6}{25}, 0\right]$. We see that the stability requirement for $\tau$ depends not only on factor $1 / \sqrt{c_{R}^{2}+c_{I}^{2}}$, but also on ratio $c_{R} / c_{I}$ as defined in formula (3.3). 


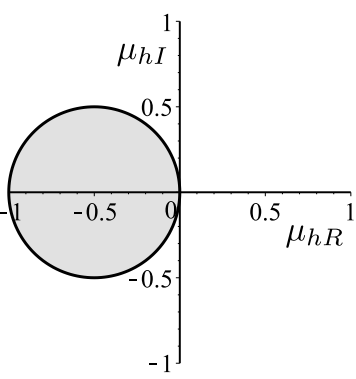

(a) parabolic equation, $c_{R}=2, c_{I}=0$

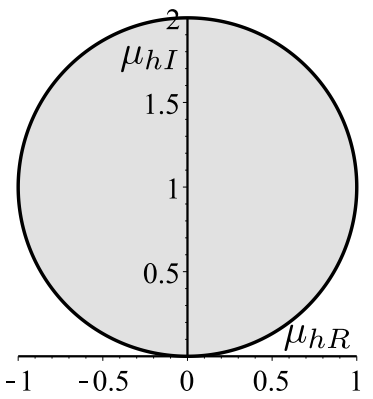

(b) Shrödinger equation, $c_{R}=0, c_{I}=1$

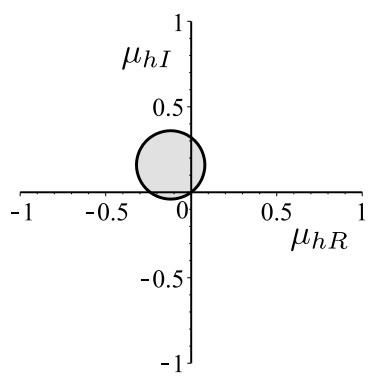

(c) Kuramoto-Tsuzuki equation, $c_{R}=3, c_{I}=4$

Figure 2. Stability regions for the explicit Euler approximation of different differential equations: a) parabolic equation, b) Schrödinger equation, c) Kuramoto-Tsuzuki equation

\section{The structure of eigenvalues for some nonlocal boundary conditions}

In this section we will present a short review on known results for the structure of eigenvalues of simple eigenvalue problem for the most simple differential operator:

$$
\frac{d^{2} u}{d x^{2}}=\lambda u, \quad 0<x<1 .
$$

We analyze the influence of nonlocal boundary conditions to the distribution of eigenvalues. Here we restrict to the case of the differential problem, but similar results are valid for discrete approximations of the diffusion operator. Then, stability results for various time integration schemes are obtained directly from the stability regions defined in the previous section.

\subsection{Nonlocal Bitsadze - Samarskii condition}

In this case we have one classical and one multi-point boundary conditions

$$
u(0)=0, \quad u(1)=\gamma u(\xi),
$$

where $\gamma, \xi \in \mathbb{R}$ and $0<\xi<1$. The eigenvalues of problem (4.1)-(4.2) depend on the parameters $\gamma$ and $\xi$. Regarding to the results presented in the paper [25] we conclude the following:

1. If $|\gamma| \leqslant 1$, then problem (4.1)-(4.2) has only real eigenvalues. All eigenvalues are real and negative for all $0<\xi<1$.

2. If $|\gamma|=\frac{1}{\xi}$, then the problem (4.1)-(4.2) has a simple eigenvalue $\lambda=0$.

3. The necessary and sufficient condition for the existence of exactly one positive eigenvalue is the inequality $\gamma>\frac{1}{\xi}$. 
4. Complex eigenvalues appear depending on the values of $\gamma$ and $\xi$ when $|\gamma|>\gamma_{1}$.

5. Multiple eigenvalues exist for some $\gamma$ and $\xi$.

\subsection{Nonlocal integral condition}

In this case we have one classical and one integral boundary conditions

$$
u(0)=0, \quad u(1)=\gamma \int_{a}^{b} u(x) d x,
$$

where $\gamma \in \mathbb{R}, a, b \in[0,1]$. The eigenvalues of the problem (4.1), (4.3) depend on parameters $\gamma, a$ and $b$. The following results are proved in $[8,19]$ :

1. For any value $\gamma$ with $a=0, b=1$ problem (4.1), (4.3) has only real eigenvalues.

2. If $\gamma>2, a=0, b=1$, then problem (4.1), (4.3) has one positive eigenvalue and the remaining eigenvalues are negative.

3. If $\gamma=2, a=0, b=1$, then problem (4.1), (4.3) has a simple eigenvalue $\lambda=0$.

4. Depending on the values of $\gamma$ with $a=\frac{1}{4}, b=\frac{3}{4}$ problem (4.1), (4.3) has also some multiple eigenvalues.

5. Problem (4.1), (4.3) with $b=1$ and any $a \in[0,1)$ has only real negative eigenvalues. One positive eigenvalue arises for $\gamma>\gamma_{0}$.

6. Problem (4.1), (4.3) with $a=0$ and any $b \in[0,1)$ depending on $\gamma$ has multiple and complex eigenvalues.

A more general differential operator

$$
\frac{d}{d x}\left(p(x) \frac{d u}{d x}\right)=\lambda u, \quad 0<x<1
$$

with nonlocal boundary conditions

$$
u(0)=\gamma_{0} \int_{0}^{1} u(x) d x, \quad u(1)=\gamma_{1} \int_{0}^{1} u(x) d x
$$

is analyzed in [22]. The eigenvalue problem (4.4)-(4.5) is solved numerically for various $p(x)$. In case $p(x) \equiv 1$ the obtained results correspond to analytic results published in [8]. In this case all eigenvalues of the discrete operator are real and negative. It is shown that one positive eigenvalue exist if $\gamma_{0}+\gamma_{1}>2$. 


\section{Numerical experiments}

In this section we present results of numerical experiments. Our main aim is to illustrate theoretical results of the previous sections.

As a test problem we solve the following linear Kuramoto-Tsuzuki type problem with an integral boundary condition:

$$
\begin{aligned}
& \frac{\partial u}{\partial t}=\left(\frac{\sqrt{2}}{2}+i \frac{\sqrt{2}}{2}\right) \frac{\partial^{2} u}{\partial x^{2}}, \\
& u(0, t)=\beta(t), \quad u(1, t)=\gamma \int_{0}^{1} u(x, t) d x, \\
& u(x, 0)=u_{0}(x), \quad 0 \leq x \leq 1,
\end{aligned}
$$

where initial condition $u_{0}$ is selected such that the integral condition is satisfied for such a function. This problem is approximated by the finite difference scheme:

$$
\begin{aligned}
& \partial_{\bar{t}} U_{j}^{n}=(1+i) \partial_{x} \partial_{\bar{x}} U_{j}^{n-1+\theta} \quad x_{j} \in D_{h}, \quad n>0, \\
& U_{0}^{n}=\beta\left(t^{n}\right), \quad U_{J}^{n}=\gamma S_{h} U^{n} .
\end{aligned}
$$

First we have used the explicit Euler scheme $\theta=0$. Then no special linear algebra solvers are required even for non local boundary conditions and all computations are done explicitly. This property of algorithms is very attractive when parallel computers are used to solve such problems.

We use the parameter $\gamma=1.582$. It was noted in the previous section, that in this case all eigenvalues of the discrete operator $A_{h}$ are real and negative. The absolute values of eigenvalues are bounded by

$$
\left|\lambda_{j}\right| \leq \frac{4}{h^{2}}
$$

From the stability interval (3.3) it follows that the explicit Euler scheme (5.1) is stable for the given Kuramoto-Tsuzuki problem if $\mu_{h R} \in[-\sqrt{2}, 0]$. Thus the time step $\tau$ should be restricted to sizes

$$
\tau \leq \frac{\sqrt{2} h^{2}}{4}
$$

This time step restriction can be compared with the well-known stability requirement for the parabolic problem (take $c_{R}=1$ and $c_{I}=0$ ) which states $\mu_{h R} \in[-2,0]$.

Computational experiments confirmed all these conclusions, the explicit Euler scheme was stable till the following restrictions on the time steps $\tau(h)$ :

$$
\tau(0.1) \leq 0.0038, \tau\left(\frac{1}{20}\right) \leq 0.0009, \tau\left(\frac{1}{40}\right) \leq 0.00022, \tau\left(\frac{1}{80}\right) \leq 0.0000553
$$

In order to show the influence of $\gamma$ to the structure of the spectrum of operators $A$ and $A_{h}$ and as a consequence to the stability of the differential 
problem, we have solved the same test problem for $\gamma=2.5$. For such a parameter the differential operator $A$ has one real positive eigenvalue. Then the corresponding eigenvector defines a growing mode of the exact PDE solution. In this case the information on the stability region of the explicit Euler scheme guarantees that we are resolving correctly all spectral modes corresponding to negative eigenvalues. The stability analysis of the growing mode is not covered by $A$-stability results, but it follows directly from the standard analysis of $\rho$-stable schemes [23].

Results of computational experiments have confirmed all these conclusions: the stability of explicit Euler scheme is observed for the same restrictions on time step as for $\gamma=1.582$. The growing mode of the solution is resolved correctly for given integration steps and the dynamics of the solution is not sensitive to the number of points used to discretize the operator $A$.

Next, we have applied the backward Euler scheme. In order to solve the obtained systems of linear equations with a matrix of special structure, we have used a superposition method to find a solution in the form $[5,6]$ :

$$
U_{j}^{n}=V_{j}^{n}+c W_{j}, \quad j=0, \ldots, J
$$

where $V$ and $W$ are solutions of linear systems with tridiagonal matrix:

$$
\begin{aligned}
& \frac{V_{j}^{n}-U_{j}^{n-1}}{\tau}=(1+i) \partial_{x} \partial_{\bar{x}} V_{j}^{n} \quad x_{j} \in D_{h}, \quad n>0, \\
& V_{0}^{n}=\beta\left(t^{n}\right), \quad V_{J}^{n}=\gamma S_{h} U^{n-1}
\end{aligned}
$$

and

$$
\begin{aligned}
& \frac{W_{j}}{\tau}=(1+i) \partial_{x} \partial_{\bar{x}} W_{j} \quad x_{j} \in D_{h}, \quad n>0, \\
& W_{0}=0, \quad W_{J}=1 .
\end{aligned}
$$

Such systems are solved efficiently by using the factorization algorithm. Coefficient $c$ is defined from the integral condition

$$
c=\left(\gamma S_{h} V^{n}-V_{J}^{n}\right) /\left(1-\gamma S_{h} W\right) .
$$

As it follows from the stability region (3.2), in the case of real eigenvalues the backward Euler scheme is stable for the given Kuramoto-Tsuzuki problem if $\mu_{h R} \notin[0, \sqrt{2}]$. Thus for $\gamma=1.582$ the backward Euler scheme is unconditionally stable and the time step $\tau$ can be selected only due to approximation accuracy requirements.

A more complicated situation arises for the case $\gamma=2.5$, when one eigenvalue $\lambda_{m}$ of discrete operator $A_{h}$ is real and positive. If time step is not sufficiently small then $\tau \lambda_{m}>\sqrt{2}$ and the numerical damping of the backward Euler scheme makes this scheme stable also for the postive eigenvalue mode. The amplitude of the discrete solution decreases and oscillates. Thus the time step $\tau$ should be reduced to satisfy the additional condition $\tau \lambda_{m}<\sqrt{2}$. This situation is illustrated in Fig. 3, where the dynamics of the solution $U_{J}^{n}$ is shown for $\tau=0.5$. 


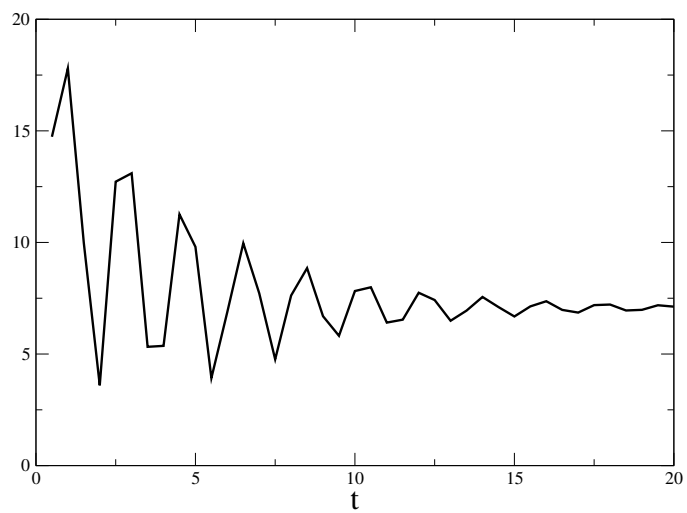

Figure 3. Dynamics of the solution $U_{J}^{n}$ of the backward Euler scheme for $\gamma=2.5$ and $\tau=0.5$

Here we note one important difference of this conditional stability requirement from the stability condition of the explicit Euler scheme. Since $\lambda_{m}$ depends very weakly on $h$, the restriction on time step $\tau$ is not depending on space step $h$.

\section{Conclusions}

A general methodology for the stability analysis of discrete approximations of nonstationary PDEs is applied to solve the Kuramoto-Tsuzuki equation, including also the Schrödinger problem. Stability regions are constructed for the explicit, backward and symmetrical Euler schemes. The obtained results are applied to solve the Kuramoto-Tsuzuki problem with a non-local integral boundary condition. Results of computational experiments are provided, they have confirmed all theoretical conclusions.

For future research, it is important to apply this methodology to investigate the stability of Schrödinger type problems with non-reflecting boundary conditions.

\section{Acknowledgement}

This research was funded by a grant (No. MIP-074/2015) from the Research Council of Lithuania.

\section{References}

[1] A. Bouziani and N. Merazga. Solution to a semilinear pseudoparabolic problem with integral condition. Elect. J. Diff. Equat., 115:1-18, 2006. 
[2] J. Cannon and A. Matheson. A numerical procedure for diffusion subject to the specification of mass. Intern. J. Engrg. Sci., 31(3):347-355, 1993. http://dx.doi.org/10.1016/0020-7225(93)90010-R.

[3] R. Ciegis. On the convergence in $\mathrm{C}$ norm of symmetric difference schemes for nonlinear evolution problems. Lith. Math. J., 32(2):187-202, 1992. http://dx.doi.org/10.1007/BF02450414.

[4] R. Čiegis, A. Štikonas, O. Štikonienè and O. Suboč. Stationary problems with nonlocal boundary conditions. Math. Model. Anal., 6(2):178-191, 2001.

[5] R. Čiegis, O. Suboč and A. Bugajev. Parallel algorithms for threedimensional parabolic and pseudoparabolic problems with different boundary conditions. Nonlinear Anal. Model. Control, 19(3):382-395, 2014. http://dx.doi.org/10.15388/NA.2014.3.5.

[6] R. Čiegis and N. Tumanova. Numerical solution of parabolic problems with nonlocal boundary conditions. Numer. Funct. Anal. Optimiz., 31(12):1318-1329, 2010. http://dx.doi.org/10.1080/01630563.2010.526734.

[7] R. Čiegis and N. Tumanova. Stability analysis of finite difference schemes for pseudoparabolic problems with nonlocal boundary conditions. Math. Model. Anal., 19(2):285-297, 2014. http://dx.doi.org/10.3846/13926292.2014.910562.

[8] R. Čiupaila, Ž. Jesevičiūtė and M. Sapagovas. On the eigenvalue problem for one-dimensional differential operator with nonlocal integral condition. Nonlinear Anal. Model. Control, 9(2):109-116, 2004.

[9] D. Dai and Y. Huang. Nonlocal boundary problems for a third-order onedimensional nonlinear pseudoparabolic equation. Nonlin. Anal., 66(1):179-191, 2007. http://dx.doi.org/10.1016/j.na.2005.11.021.

[10] M. Dehghan. Efficient techniques for the second-order parabolic equation subject to nonlocal specifications. Appl. Numer. Math., 52(1):39-62, 2005. http://dx.doi.org/10.1016/j.apnum.2004.02.002.

[11] G. Fairweather and J.C. Lopez-Marcos. Galerkin methods for a semilinear parabolic problem with nonlocal boundary conditions. Advances in Comput. Mathm., 6:243-262, 1996.

[12] A.V. Gulin, V.A. Morozova and N.S. Udovichenko. Stability criterion for a family of nonlocal difference schemes. Diff. Equat., 46(7):2716-2732, 2010. http://dx.doi.org/10.1134/S0012266110070050.

[13] W. Hundsdorfer and J.G. Verwer. Numerical Solution of Time-Dependent Advection-Difusion-Reaction Equations, volume 33 of Springer Series in Computational Mathematics. Springer, Berlin, Heidelberg, New York, Tokyo, 2003. http://dx.doi.org/10.1007/978-3-662-09017-6.

[14] J. Jachimavičienè, Ž. Jasevičiūtè and M. Sapagovas. The stability of finite-difference schemes for a pseudoparabolic equation with nonlocal conditions. Numer. Funct. Anal. Optimiz., 30(9):988-1001, 2009. http://dx.doi.org/10.1080/01630560903405412.

[15] Ž. Jesevičiūtė and M. Sapagovas. On the stability of finite-difference schemes for parabolic equations subject to integral conditions with applications to thermoelasticity. Computational Methods in Applied Mathematics, 8(4):360-373, 2008.

[16] Y. Kuramoto. Chemical Oscillations, Waves, and Turbulence. Springer-Verlag, Berlin Heidelberg NewYork Tokyo, 1984. 
[17] Yoshiki Kuramoto and Toshio Tsuzuki. On the formation of dissipative structures in reaction-diffusion systems reductive perturbation approach. Prog. Theor. Phys., 54(3):687-699, 1975. http://dx.doi.org/10.1143/PTP.54.687.

[18] J. Martin-Vaquero and J. Vigo-Aguiar. On the numerical solution of the heat conduction equations subject to nonlocal conditions. Appl. Numer. Math., 59(10):2507-2514, 2009. http://dx.doi.org/10.1016/j.apnum.2009.05.007.

[19] S. Pečiulytè, O. Štikonienè and A. Štikonas. Sturm-Liouville problem for stationary differential operator with nonlocal integral boundary condition. Math. Model. Anal., 10(4):377-392, 2005.

[20] M. Radziunas and F. Ivanauskas. The stability conditions of finite difference schemes for Schrodinger, Kuramoto-Tszuki and heat equations. Math. Model. Anal., 3(1):177-194, 1998.

[21] M. Radziunas and F. Ivanauskas. The convergence and stability of splitting finite-difference schemes for nonlinear evolutionary equations. Lith. Math. J., 45(3):334-352, 2005. http://dx.doi.org/10.1007/s10986-005-0035-2.

[22] S. Sajavičius and M. Sapagovas. Numerical analysis of the eigenvalue problem for one-dimensional differential operator with nonlocal integral conditions. Nonlinear Anal. Model. Control, 14(1):115-122, 2009.

[23] A.A. Samarskii. The Theory of Difference Schemes. Marcel Dekker, Inc., New York-Basel, 2001. http://dx.doi.org/10.1201/9780203908518.

[24] M. Sapagovas. On the stability of finite-difference scheme for nonlocal parabolic boundary-value problems. Lituanian Math. J., 48(3):339-356, 2008. http://dx.doi.org/10.1007/s10986-008-9017-5.

[25] M.P. Sapagovas and A.D. Štikonas. On the structure of the spectrum of a differential operator with nonlocal condition. Differential Equations, 41(7):1010-1018, 2005 .

[26] Zhi-Zhong Sun and Qi-Ding Zhu. On Tsertsvadze's difference scheme for the Kuramoto-Tsuzuki equation. J. Comput. Appl. Math., 98(2):289-304, 1998. http://dx.doi.org/10.1016/S0377-0427(98)00135-6.

[27] G. Z. Tsertsvadze. On the convergence of difference schemes for the KuramotoTsuzuki equation and reaction-diffusion type systems. Zh. Vychisl. Mat.Mat. Fiz., 31(5):698-707, 1991.

[28] T. Wang and B. Guo. A robust semi-explicit difference scheme for the $\mathrm{Ku}$ ramoto - Tsuzuki equation. J. Comput. Appl. Math., 233(4):878-888, 2009. http://dx.doi.org/10.1016/j.cam.2009.07.058.

[29] Y. Yang. Convergence of the pseudospectral method for the GinzburgLandau equation. J. Math. Anal. Appl., 147(2):556-568, 1990. http://dx.doi.org/10.1016/0022-247X(90)90370-U. 